\title{
ESTUDIO DE LA DEMANDA EXISTENTE EN TORNO AL OLEOTURISMO. EL CASO DE ANDALUCÍA
}

\author{
Francisco Orgaz Agüera* \\ Universidad Tecnológica de Santiago. República Dominicana \\ Salvador Moral Cuadra** \\ Universidad de Córdoba \\ Tomás López-Guzmán*** \\ Universidad de Córdoba \\ Pablo Cañero Morales**** \\ Universidad de Córdoba
}

\section{RESUMEN}

El oleoturismo se desarrolla en el medio rural donde se refleja una determinada cultura en relación con el aceite de oliva. El objetivo de este artículo es analizar el perfil sociodemográfico, las actividades, las motivaciones, las valoraciones y los niveles de satisfacción que tienen con el viaje los oleoturistas en varias zonas geográficas de Andalucía. Para ello se realizan encuestas a la demanda. Los resultados muestran que destacan visitantes de $60 \mathrm{o}$ más años, con una renta media-alta, estudios universitarios y extranjeros. Las motivaciones son visitar la zona geográfica, aprender sobre el mundo del aceite y comer y beber productos de la zona. Lo más valorado es la hospitalidad y la conservación del entorno. La mayoría están satisfechos con el viaje realizado.

Palabras clave: Oleoturismo, Aceite de oliva, Demanda, Satisfacción, Andalucía.

Recibido: 14 de enero de 2015

Devuelto para su revisión: 5 de junio de 2015

Aceptado: 8 de julio de 2015

* Universidad Tecnológica de Santiago, UTESA. Av. Estrella Sadhalá, 75.51000 SANTIAGO (República Dominicana).E-mail: franorgaz@utesa.edu

** Departamento de Estadística, Econometría, Investigación Operativa, Organización de Empresas y Economía Aplicada. Universidad de Córdoba. CÓRDOBA (España).E-mail: 162mocus@uco.es

*** Universidad de Córdoba. Área de Economía Aplicada. Facultad de Ciencias del Trabajo. C/ Adarve, 30. 14071 CÓRDOBA (España).E-mail: tomas.lopez@uco.es

**** Departamento de Estadística, Econometría, Investigación Operativa, Organización de Empresas y Economía Aplicada. Universidad de Córdoba. CÓRDOBA (España).E-mail: u72camop@uco.es 


\title{
Study of demand around the olive oil tourism. The case of Andalusia
}

\begin{abstract}
The olive oil tourism develops in rural areas which reflect a particular culture in relation to olive oil. The purpose of this study is to analyze the demographic profile, activities, motivations, valuations and levels of satisfaction with the trip in various geographical areas of Andalusia's olive tourism. There has been a methodology based on surveys. The results show that visitors with 60 years or olders, with a medium-high income, university studies and foreign. The motivations are visiting the geographical area, learn about the world of oil and eat and drink local products. The most valued is the hospitality and environmental conservation. Most tourists are satisfied with the trip.
\end{abstract}

Keywords: Olive tourism, Olive oil, Demand, Satisfaction, Andalusia.

\section{INTRODUCCIÓN}

El turismo se conforma como un sector económico que ha experimentado un cambio de tendencia, debido a que se ha pasado de un turismo de masas a uno más individualizado, puesto que han aparecido turistas más exigentes con nuevas actitudes y necesidades (Cracolici y Nijkamp, 2009). De esta forma, la actividad turística requiere de un tratamiento específico por la variedad en las interrelaciones que se generan entre todos los elementos que conforman la oferta y la demanda, debido a que el turista interactúa con las organizaciones prestatarias del servicio, el entorno y el contexto en el que se desarrolla el servicio, dependiendo su grado de satisfacción de las diferentes características físicas, humanas u organizativas del servicio contratado. En este sentido, el turismo opera como fuente de oportunidades para la modernización social, económica y cultural de una región determinada. Así, los productos gastronómicos son un elemento cultural que fomenta el desarrollo sostenible de un área geográfica (López-Guzmán y Sánchez-Cañizares, 2012) a través de la potencialización de las tradiciones y costumbres culturales de la población local, que implica un creciente interés por el turismo realizado en entornos rurales, debido al incremento tanto de la demanda turística como también de la oferta de infraestructura y servicios turísticos (Pulina et al., 2006).

De esta manera, el turismo gastronómico está experimentado un gran crecimiento en la literatura científica, debido a que gran parte de la diversión de los viajes se ha asociado a la comida local de un destino turístico (Amuquandoh y Asafo-Adjei, 2013), siendo esto un importante elemento de atracción para determinadas regiones y/o países (Sim, 2009), puesto que el deseo de viajar y degustar comidas únicas y auténticas representa un fenómeno emergente en la industria del turismo (Smith y Costello, 2009). Dentro del mundo de la gastronomía, han aparecido otras actividades que se encuadran dentro de lo que se conoce como turismo temático, destacando el propio turismo gastronómico, enoturismo o el oleoturismo (López-Guzmán et al., 2013). Centrándonos en el oleoturismo, a la hora de desarrollar esta actividad se debe tener en cuenta el territorio como uno de los factores 
claves que determinan su oferta y su demanda (Ruiz Guerra et al., 2011), teniendo en cuenta que el medio rural es parte integral de la experiencia, puesto que el destino incluye paisajes y clima, y tal y como indica Aybar (2004), un paisaje rural implica también un estilo de vida que refleja una determinada cultura y que contribuye a crear una imagen turística. Por estas razones, esta modalidad turística está incluida dentro del turismo rural (Alonso, 2010; Alonso y Northcote, 2010; Millán Vázquez de la Torre et al., 2012), aunque Ruiz Guerra (2010) considera que el oleoturismo es una manifestación del agroturismo, turismo cultural y turismo de salud.

En este sentido, el objetivo de esta investigación es analizar el perfil sociodemográfico de los visitantes, las actividades que realizan, las motivaciones para realizar la visita, las valoraciones con el destino turístico y los niveles de satisfacción que tienen con el viaje. El estudio se realiza en varias zonas geográficas de la región de Andalucía, siendo esta región el principal productor de aceite de oliva con certificado de calidad en España (De Salvo et al., 2013), situándose en el primer lugar en explotaciones y producción, lo que favorece el fomento de esta tipología de turismo en esta zona geográfica. De esta forma, se pretende fortalecer las investigaciones en el campo del oleoturismo, que aún tienen carencias debido a que estamos ante una tipología de turismo reciente (Alonso y Northcote, 2010), y por tanto, requiere una adecuada metodología para su análisis (Campón-Cerro et al., 2014). Por esta razón, es necesario incrementar las investigaciones académicas en temas relacionados con la cultura oleícola (Ruiz Guerra, 2010). Para cumplir estos objetivos, este artículo se ha estructurado, tras esta introducción, en un segundo apartado que trata una revisión de la literatura sobre el tema de estudio. Posteriormente, en un tercer apartado se describe el área geográfica donde se desarrolla esta investigación. En un cuarto apartado se muestra la metodología empleada en este estudio, para después, en un quinto apartado desarrollar los principales resultados del estudio. El artículo termina con las conclusiones y la bibliografía utilizada en la investigación.

\section{REVISIÓN DE LA LITERATURA}

El término oleoturismo apareció como una línea de comercialización directa de los productos oleícolas a los consumidores, generando otras fuentes de negocios (Alonso y Krajsic, 2013) en línea con lo sucedido con otras tipologías encuadradas dentro del turismo temático (Quand y Wang, 2004; Okumus et al., 2007; Velioka et al., 2013). De esta forma, las primeras investigaciones sobre el oleoturismo se realizaron en Australia (Alonso, 2010; Alonso y Northcote, 2010; Northcote y Alonso, 2011), ampliándose posteriormente al continente europeo, sobre todo a regiones de Italia y España (Ruiz Guerra, 2010; LópezGuzmán y González-Fernández, 2011; Murgado, 2013; De Salvo et. al., 2013; Millán Vázquez de la Torre et al., 2014; Campón-Cerro et al., 2014). Así, el término que relaciona el turismo con el aceite de oliva ha sido llamado, además de oleoturismo, de diversas formas en inglés, tales como agrotourism, olive oil tourism y olive-based agritourism. Aunque, según Murphy (1985), farm tourism apareció en las primeras investigaciones sobre esta temática como forma de relación entre los trabajos dentro la actividad agrícola y el turismo. Así, esta actividad turística podría suponer un reforzamiento de la imagen del aceite de oliva y un aumento del valor añadido del mismo (Alonso y Northcote, 2010). En 
este sentido, Telfer y Wall (1996) afirman que existe una estrecha relación entre el sector turismo y el agropecuario, debido a que, y siguiendo a Socher y Tschurtschenthaler (1994), tanto la agricultura como la ganadería sirven, además de alimentar a los visitantes, para confeccionar productos intangibles.

La práctica del oleoturismo adquiere dentro del turismo industrial rural una alta potencialidad, consistiendo esta actividad en la organización de visitas a los centros cooperativos en los que se procesa el producto agrario para transformarlo en alimento, puesto que un alto porcentaje de las almazaras dedicadas a la producción de aceite de oliva son cooperativas situadas en el medio rural (Moral Cuadra et al., 2014). Los principales visitantes a estas áreas geográficas proceden de los viajes de autobús, grupos de estudiantes y grupos de comunidades (Northcote y Alonso, 2011). Aunque, actualmente están poco desarrolladas las rutas turísticas debido a la existencia de un escaso número de actividades turísticas y al bajo desarrollo en términos de marketing y management (Murgado, 2013). Así, es importante la localización geográfica para realizar esta tipología turística, puesto que existe una ventaja competitiva importante en aquellas zonas que se encuentran cerca de las principales rutas turísticas, aunque también se debe tener en cuenta los niveles de asociación entre las empresas privadas y los demás stakeholders locales y regionales, así como los servicios turísticos que existan en el área geográfica (Northcote y Alonso, 2011).

Entre las actividades relacionadas con el oleoturismo, encontramos la experiencia sensorial (Getz, 2000), donde el turista experimenta el placer del sabor, olor, tacto, vista y sonido, teniendo como principales componentes la visita a las almazaras y campos de cultivo milenarios, así como la degustación de aceite de oliva y platos derivados, o la compra de productos (Brunori y Rossi, 2000), por lo que esta actividad turística puede aportar un gran valor paisajístico, cultural y medioambiental a un área geográfica determinada, y a través de la actividad turística puede contribuir a promover la conservación (Alonso y Krajsic, 2013). En este sentido, hay que profundizar en la eliminación de barreras que suelen considerarse en este tipo de actividades turísticas, como pueden ser las cognitivas (no tienen valores estéticos, no es interesante, no es elegante), económicas (es más rentable vender la maquinaria como chatarra, para enajenar el área o dedicar el edificio a otras ocupaciones), legales o administrativas de los obstáculos (poco claro en las actuaciones a realizar) y las físicas (la distancia de algunas instalaciones con respecto a las tradicionales rutas turísticas) (Capel, 1996). De esta manera, para algunos autores (Clark y Chabrel, 2007; Woodland y Acott, 2007), la gastronomía local puede generar importantes beneficios tanto a los residentes como a los turistas, debido a que estos productos pueden generar diversas implicaciones para la sostenibilidad de un destino, fomentando además la cultural local (Kivela y Crotts, 2006). Así, el consumo de alimentos de producción local y la utilización de productos locales en la preparación de menús para turistas fomenta la reducción de las importaciones y la fuga de los ingresos en la economía local por la actividad turística (Torres, 2002).

Entre los factores que determinan la elección de visitar destinos para realizar actividades relacionadas con la gastronomía, están las características del lugar de origen, la religión, el sexo, la educación, el nivel de vida y la disponibilidad de alimentos en el destino (Woolcott et al., 1981; Amuquandoh y Asafo-Adjei, 2013). Estos elementos influyen también en las motivaciones de los turistas a la hora de visitar un determinado lugar. En 
este sentido, McIntosh et al. (1995) identifica cuatro motivaciones para visitar un lugar: físicas, culturales, interpersonales y de estatus y prestigio. De esta manera, Bonifacio (2003) identifica cinco motivaciones de los visitantes que practican el turismo en zonas donde se producen productos gastronómicos locales; la primera se refiere a la ansiedad de los visitantes que quieren celebrar con comida y bebida un día de fiesta; las segunda viene por la necesidad de mostrar la distinción, la riqueza, y el individualismo; la tercera hace referencia a la curiosidad y el deseo de conocimiento y descubrimiento para mostrar la educación y la superioridad sobre los demás; la cuarta viene marcada por la necesidad de sentirse conectado a un lugar determinado, donde localiza sus propias raíces; la quinta y última, el requisito de placer sensorial y táctil a través de una experiencia gastronómica.

Siguiendo a Murgado (2013), entre los principales problemas para el desarrollo del oleoturismo encontramos la falta de cooperación entre los agentes implicados en la oferta de productos turísticos; la inexperiencia y la falta de capacitación del personal entre los prestadores de servicios turísticos en relación al aceite de oliva; la deficiente infraestructura de negocios; la falta de productos turísticos atractivos en el mercado para desencadenar la demanda de aceite de oliva relacionadas con el turismo; y los pobres modelos de marketing para promocionar y comercializar esta actividad turística. De esta manera y de acuerdo con Hillel et al. (2013), las rutas de turismo relacionada con elementos gastronómicos pueden ser un componente más de un producto turístico, y cada una tendrá un valor único según las características históricas y culturales del destino geográfico (Bruwer, 2003). Así, algunos autores (Correia et al., 2004; Tomljenovic y Getz, 2009) resaltan la necesidad de establecer relaciones entre los stakeholders privados y públicos para poner en marcha estos productos turísticos en un área geográfica determinada.

Por tanto, el oleoturismo a través de una correcta planificación genera beneficios para el desarrollo rural, marco en el que generalmente se desarrolla esta actividad turística. Entre los principales beneficios destaca la creación de nuevas empresas y empleos; el incremento de los impactos positivos en el área geográfica a partir de nuevas formas de explotación del olivar y del aceite de oliva; la aparición de nuevas sinergias con otros sectores, haciendo que se generen nuevos beneficios económicos para las comunidades rurales locales; la conservación de las técnicas tradicionales de producción del aceite de oliva; y, el desarrollo del turismo gastronómico y turismo industrial, que a su vez pueden fomentar el desarrollo socioeconómico y sostenible en el destino geográfico.

\section{DESCRIPCIÓN DEL ÁREA GEOGRÁFICA}

Siguiendo al Consejo Oleícola Internacional (2013), España es el principal país en relación a la producción mundial de aceite de oliva, seguida de Italia y Grecia. En este sentido, España produce más del $60 \%$ de la producción mundial de aceite de oliva, con la excepción de la campaña 2012/13 donde la producción fue muy baja debido a las condiciones climatológicas. Así, y dentro de España, la región de Andalucía suele aportar algo más del $80 \%$ de la producción total y, al mismo tiempo, más del $60 \%$ de esa producción procede de dos provincias, Jaén y Córdoba. Es decir, estas dos provincias aportan anualmente una cifra cercana al $35 \%$ de la producción mundial de aceite de oliva (Moral Cuadra et al., 2014). Por tanto, las actividades agrícolas relacionadas con el aceite de oliva 
tienen una gran importancia para Andalucía, apareciendo sinergias entre la producción de aceite de oliva y la actividad turística. En relación a otros productos agrícolas, ya existen casos de sinergias en esta región, como es el caso del vino (Vargas Sánchez et al., 2012; López-Guzmán et al., 2014) o la comida (Morales Fernández, 2012; Millán Vázquez de la Torre et al., 2014).

España tiene repartida por su geografía un total de 31 Denominaciones de Origen Protegidas (DOP) relacionadas con el aceite de oliva y reconocidas por el Ministerio de Agricultura, Alimentación y Medio Ambiente (MAGRAMA). Según Hinojosa-Rodríguez et al. (2014), las DOP permiten reconocer la calidad del producto, basado en sus propias características y donde su calidad se diferencia dependiendo de cada área geográfica donde se produzca. Así, 12 de las 31 DOP se localizan en la región andaluza (Mapa 1), y siete de ellas se encuentran en las provincias de Jaén y Córdoba, que son las áreas geográficas donde se realiza esta investigación.

\section{Mapa 1 \\ DENOMINACIONES DE ORIGEN \\ PROTEGIDAS LOCALIZADAS EN ANDALUCÍA}

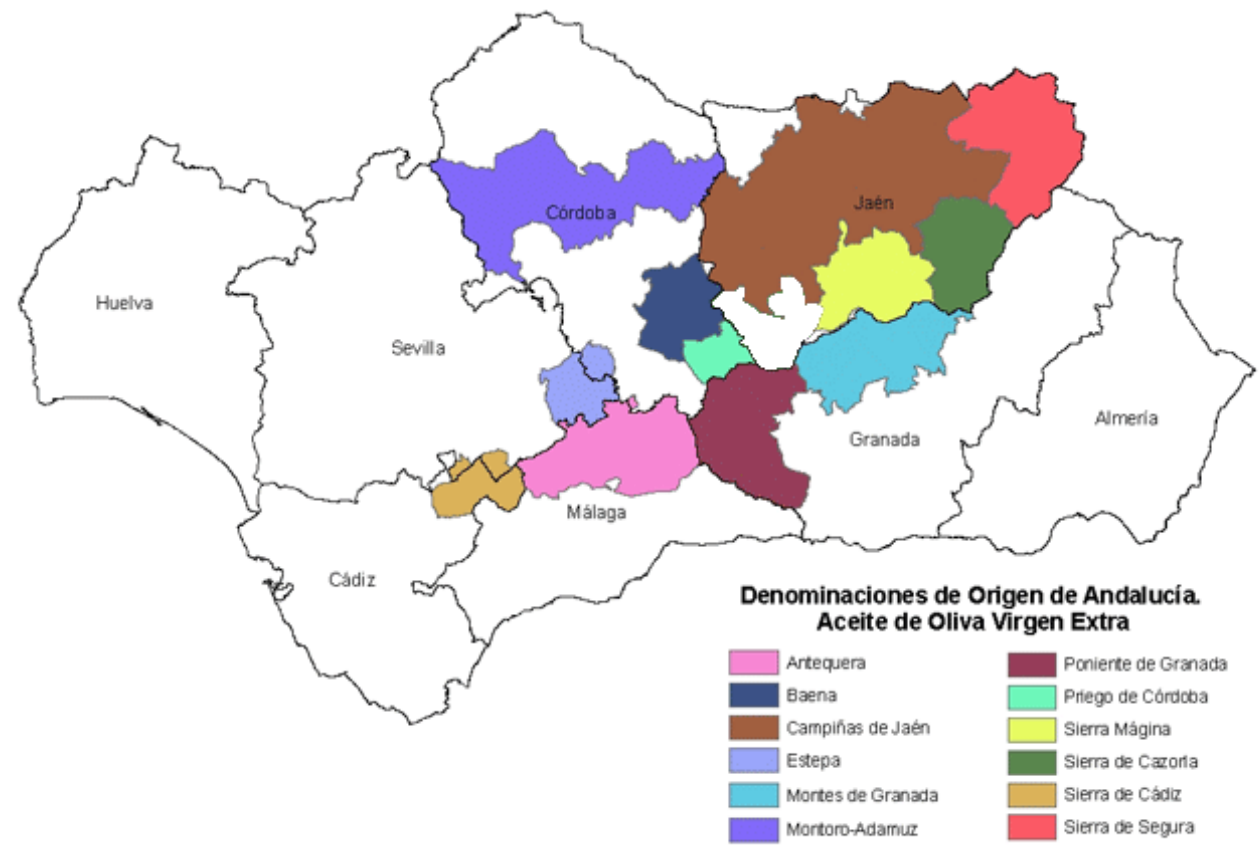

Fuente: www.bedri.es

Siguiendo el Mapa 1, en Jaén se localizan las DOP de Sierra Mágina, Sierra de Segura, Sierra de Cazorla; y en la provincia de Córdoba se encuentran las DOP de Baena, Lucena, Priego de Córdoba y Montoro-Adamuz. En este aspecto, en la provincia de Jaén existe 
una ruta denominada Oleoturismo (www.oleoturismo.com), donde se relacionan aspectos de la producción oleícola con la cultura, patrimonio, innovación, economía, estrategias de desarrollo y medio ambiente (Aybar, 2004). Referente a la provincia de Córdoba, existen ocho rutas diferentes sobre oleoturismo que abarcan las diferentes DOP de la provincia. De esta manera, y de acuerdo a Murgado (2013), ambas provincia manifiestan con sus propuestas el potencial para desarrollar el oleoturismo en Andalucía y España. Así, y teniendo en cuenta que la población mundial cada vez está más concienciada con el desarrollo sostenible y el respeto hacia la naturaleza y cultura local, el oleoturismo se configura como una práctica turística sostenible para Andalucía, máxime cuando con el desarrollo de las DOP se ha mejorado la demanda de estos productos, debido a la alta calidad, seguridad de la alimentación, métodos de producción en el lugar de origen, la disponibilidad y el ratio de calidad-precio favorable (Aramyan et al., 2006). Aunque en Andalucía existen deficiencias a la hora de poner en marcha el oleoturismo, debido a que la infraestructura de alojamientos (hoteles rurales, casas rurales, etc.) aún son escasos, no existiendo tampoco una visión estratégica óptima para desarrollar este tipo de turismo (Moral Cuadra et al., 2014), y muchas de las almazaras y museos de olivo no se encuentran actualmente preparados para recibir flujos de visitantes (Millán Vázquez de la Torre et al., 2011). Por otro lado, si se realizan diversos eventos de forma anual en estos destinos que fomentan el oleoturismo, con la misión de mejorar el valor de los aceites de oliva vírgenes extras de mayor calidad y estimular a los productores a obtener y comercializar los mejores aceites. Tal es el caso de a Cata-Concurso de Aceites de Oliva Virgen Extra o Oleotour Jaén, que se celebran en Jaén cada año (Diputación de Jaén, 2015).

\section{METODOLOGÍA}

El trabajo de campo de esta investigación tiene como objetivo conocer diferentes aspectos relacionados con el turista interesado en el conocimiento de la producción del aceite de oliva. De esta forma, se ha analizado el perfil sociodemográfico de los visitantes, las actividades que realizan, las motivaciones para realizar la visita, las valoraciones de los principales elementos del destino y los niveles de satisfacción generales que tienen con el viaje. Se ha utilizado una metodología cuantitativa, basada en la encuesta, y dirigida a los visitantes que llegan a la región de Andalucía, en concreto a las zonas de Jaén y Córdoba, las principales provincias en producción de aceite de oliva de España. Se ha utilizado un muestreo de conveniencia, comúnmente utilizado en este tipo de investigaciones, donde el público objetivo está disponible para ser encuestados en un espacio y tiempo determinado (Finn et al., 2000). La estructura seguida en el cuestionario se ha basado en estudios previos (Alonso y Nortcote, 2010; Molina Moreno et al., 2011; De Salvo et al., 2013) y responde a tres grupos de variables analizadas durante la visita del turista (Poria et al., 2003): características sociodemográficas del turista encuestado, motivaciones para visitar la zona geográfica y valoración por parte de los turistas sobre diferentes variables relacionadas con el destino.

El trabajo de campo se ha desarrollado entre los meses de abril y octubre de 2014. Para su realización, se eligieron seis puntos estratégicos en las zonas oleícolas más importantes de Andalucía. Esos puntos fueron dos almazaras, dos museos del aceite de oliva y dos 
centros de interpretación, localizados en las provincias de Jaén y Córdoba y que corresponden, como se viene mencionando, con las DOP con mayor producción anual de aceite de oliva en España. Los visitantes rellenaron el cuestionario con total independencia, aunque los encuestadores estaban presentes por sí tenían algún tipo de dificultad para rellenarla. La encuesta se distribuyó en cuatro idiomas (español, inglés, francés y alemán), siendo totalmente anónima. Anteriormente al desarrollo del trabajo de campo, se efectuó un pretest de 30 encuestas para detectar posibles desviaciones y errores. Se obtuvo un total de 414 encuestas válidas (tabla 1). El número de ítems de cada encuesta fue de 19, estando estructurado en preguntas basadas en una escala de Likert de 5 puntos para valorar la opinión del visitante, en respuestas sí/no para obtener la percepción sobre determinados aspectos relacionados con el área geográfica y cuestiones cerradas y abiertas donde los turistas expresan su experiencia oleoturística.

\section{Tabla 1 \\ FICHA TÉCNICA DE LA INVESTIGACIÓN}

\begin{tabular}{|r|l|}
\hline Muestra & 414 encuestas válidas \\
\hline Procedimiento & Muestreo por conveniencia \\
\hline Temporalidad & Abril-octubre de 2014 \\
\hline Lugar de realización & Seis puntos de encuestación en las provincias de Jaén y Córdoba \\
\hline Control de la muestra & Realización y supervisión del trabajo de campo por los autores \\
\hline
\end{tabular}

Fuente: elaboración propia.

Teniendo en cuenta que existen pocas investigación en relación al oleoturismo, no se dispone en la región de Andalucía de un dato fiable sobre el número de turistas en este segmento, y por tanto, no se puede determinar una población objetiva. El índice de fiabilidad según el alfa de Cronbach fue de 0,747. En este sentido, aunque hay autores que consideran aceptable una escala si su alfa de Cronbach está por encima de 0,7 (Nunnally y Berstein, 1994), hay otros investigadores que han sugerido que son aceptables valores superiores a 0,6 (Black y Porter, 1996; Petrick y Backman, 2002). En cualquiera de los casos, nuestro estudio supera los valores mínimos aceptados en la literatura científica. Los datos de esta investigación se han tabulado y analizado utilizando el sistema estadísticos IBM SPSS 19.

\section{ANÁLISIS DE LOS RESULTADOS Y DISCUSIÓN}

La tabla 2 muestra las características sociodemográficas de los oleoturistas, observándose las variables sexo, edad, país de procedencia, categoría profesional, nivel de renta y nivel de educación. En este aspecto, destacan las mujeres, con edades de 60 o más años, extranjeros, jubilados, con una renta media-alta y con educación universitaria. 
Tabla 2

PERFIL SOCIODEMOGRÁFICO DE LOS OLEOTURISTAS

\begin{tabular}{|c|c|c|c|}
\hline Variables & Porcentaje & Variables & Porcentaje \\
\hline $\operatorname{Sexo}(N=394)$ & $\begin{array}{l}43,1 \% \\
56,9 \%\end{array}$ & \begin{tabular}{|r|} 
Nivel de estudios $(N=388)$ \\
Educación primaria \\
Educación secundaria \\
Educación universitaria \\
\end{tabular} & $\begin{array}{l}12,6 \% \\
35,1 \% \\
52,3 \%\end{array}$ \\
\hline $\begin{array}{l}\text { Edad }(N=391) \\
\qquad \begin{array}{r}\text { Menos de } 30 \text { años } \\
30-39 \text { años } \\
40-49 \text { años } \\
50-59 \text { años } \\
60 \text { años o más }\end{array}\end{array}$ & $\begin{array}{c}14,6 \% \\
5,1 \% \\
9,7 \% \\
16,9 \% \\
53,7 \%\end{array}$ & $\begin{array}{r}\text { Categoría profesional }(N=386) \\
\text { Profesional liberal } \\
\text { Asalariado } \\
\text { Funcionario } \\
\text { Estudiante } \\
\text { Ama de casa } \\
\text { Jubilado }\end{array}$ & $\begin{array}{c}16,1 \% \\
13,7 \% \\
10,1 \% \\
13,0 \% \\
6,7 \% \\
40,4 \% \\
\end{array}$ \\
\hline $\begin{array}{r}\text { Renta }(N=330) \\
\text { Menos de } 700 € \\
\text { De } 701-1.000 € \\
\text { De } 1.001-1.500 € \\
\text { De } 1.501-2.500 € \\
\text { Más de } 2.500 €\end{array}$ & $\begin{array}{c}6,1 \% \\
6,3 \% \\
23,7 \% \\
32,7 \% \\
31,2 \%\end{array}$ & $\begin{array}{r}\text { País de procedencia }(N=401) \\
\text { España } \\
\text { Francia } \\
\text { Reino Unido } \\
\text { Alemania } \\
\text { Otros }\end{array}$ & $\begin{array}{c}43,1 \% \\
28,2 \% \\
11,7 \% \\
6,2 \% \\
10,8 \%\end{array}$ \\
\hline
\end{tabular}

Fuente: elaboración propia.

Siguiendo la tabla 2, se encontró asociación entre el nivel de renta y sexo (Chi-cuadrado de Pearson $=14,432 ; \mathrm{p}=0,006)$, nivel de renta y edad (Chi-cuadrado de Pearson $=36,273 ; \mathrm{p}=0,014$ ), nivel de renta y estudios (Chi-cuadrado de Pearson $=76,533 ; \mathrm{p}=$ 0,000 ), nivel de renta y categoría profesional (Chi-cuadrado de Pearson $=49,976 ; \mathrm{p}=$ $0,000)$ y nivel de renta y país de procedencia (Chi-cuadrado de Pearson $=98,502 ; \mathrm{p}=$ $0,000)$.

El 68,1\% de los oleoturistas es la primera vez que visitan esta zona geográfica. Así, el 75,6\% de los visitantes son turistas, siendo el restante $24,4 \%$ excursionistas que hacen viajes de un día para visitar estos recursos turísticos relacionados con el aceite de oliva. Del porcentaje de turistas que visita estos destinos, el 47,1\% permanece en la zona geográfica entre 3 y 7 días. El 40,8\% viaja con compañeros de trabajo o amigos y el 34,6\% con pareja, apareciendo pocos turistas individuales en estos lugares, confirmando esto el resultado obtenido por Northcote y Alonso (2012). El 33,1\% han elegido esta zona geográfica como destino turístico por experiencia propia, destacando también un $24,6 \%$ que eligieron estos recursos por recomendación de amigos y familiares.

Entre las actividades que han realizado durante el viaje, en la tabla 3 se puede observar que destaca el turismo cultural $(74,6 \%)$. Esto puede provenir a que las principales zonas de producción de aceite de oliva de esta investigación se localizan cerca de ciudades que 
son Patrimonio de la Humanidad (Córdoba, Úbeda y Baeza), y cerca de la provincia de Málaga, destino muy consolidado en turismo de sol y playa (López-Guzmán y GonzálezFernández, 2011), pero que como tal, necesita de ofertas complementarias para diversificar la oferta principal del destino.

Tabla 3

ACTIVIDADES TURÍSTICAS REALIZADAS POR LOS VISITANTES

\begin{tabular}{|c|c|}
\hline Actividad Turística & Porcentaje $*$ \\
\hline Turismo cultural & $74,6 \%$ \\
\hline Turismo rural & $35,0 \%$ \\
\hline Oleoturismo & $22,2 \%$ \\
\hline Turismo gastronómico & $18,3 \%$ \\
\hline Otros & $4,9 \%$ \\
\hline
\end{tabular}

Fuente: Elaboración propia. *La suma total supera el 100\% porque la respuesta es simultánea.

Profundizando más las principales motivaciones para realizar las actividades durante este viaje en esta zona geográfica, se han analizado a través de una escala de Likert de 5 puntos, siendo 1 muy poco y 5 mucho, y con índice de fiabilidad según el alfa de Cronbach de 0,767 .

Tabla 4

MOTIVACIONES DE LOS VISITANTES

\begin{tabular}{|r|c|}
\hline Motivaciones & Media \\
\hline Visitar la zona geográfica & 3,94 \\
\hline Aprender sobre el mundo del aceite & 3,74 \\
\hline Comer y beber productos de la zona & 3,53 \\
\hline Entretenimiento & 3,46 \\
\hline Relajación & 3,13 \\
\hline Pasar un día fuera & 3,06 \\
\hline Catar diferentes aceites de oliva & 2,89 \\
\hline Comprar aceite de oliva & 2,62 \\
\hline Visitar amigos y familiares & 1,81 \\
\hline Motivos de trabajo & 1,61 \\
\hline
\end{tabular}

Fuente: elaboración propia.

De esta forma, y siguiendo la tabla 4, se puede observar que destaca visitar la zona geográfica (3,94 puntos sobre 5), aprender sobre el mundo del aceite (3,74 puntos sobre 5) 
y comer y beber productos de la zona (3,53 puntos sobre 5). En cuanto al aprendizaje del mundo del aceite se considera una de las razones más importantes que refuerza la experiencia del visitante (Alonso y Krajsic, 2013) junto con la degustación de los productos gastronómicos típicos de la zona, confirmando así que los alimentos y bebidas son unas de las preferencias de los visitantes (Amuquandoh y Asafo-Adjei, 2013) debido a que los turistas cuando viajan consumen platos gastronómicos de la región y bebidas locales, prefiriendo estos alimentos sobre otros (Rozin y Volhmecke, 1986; Chang et al., 2010). Por otro lado, resulta significativo el resultado de la poca valoración que dan los turistas encuestados a la compra de aceite en estos viajes, considerando que este resultado tiene su origen en el problema del traslado del aceite de oliva en los aviones debido a que los niveles de intención de compra son bastante menores entre los extranjeros que entre los españoles, los cuáles se desplazan habitualmente en autobús, siendo estos resultados similares a los de otras investigaciones del campo del enoturismo en Andalucía (LópezGuzmán et al., 2014).

Las principales valoraciones de los visitantes hacia el destino turístico también se han analizado a través de una escala de Likert de 5 puntos, siendo 1 muy poco y 5 mucho, y con un índice de fiabilidad según el alfa de Cronbach de 0,899.

Tabla 5

VALORACIONES DE LOS OLEOTURISTAS

\begin{tabular}{|r|c|r|c|}
\hline \multicolumn{1}{|c|}{ Aspecto a valorar } & Media & Aspecto a valorar & Media \\
\hline Hospitalidad & 4,18 & Almazara & 3,65 \\
\hline Alimentación & 3,89 & Información & 3,51 \\
\hline Conservación & 3,83 & Precio del viaje & 3,50 \\
\hline Restauración & 3,82 & Ecología & 3,49 \\
\hline Actividades culturales & 3,81 & Comunicación & 3,34 \\
\hline Limpieza & 3,80 & Telecomunicaciones & 3,32 \\
\hline Seguridad ciudadana & 3,69 & Zonas comerciales & 3,01 \\
\hline Alojamiento & 3,69 & Actividades deportivas & 2,55 \\
\hline
\end{tabular}

Fuente: elaboración propia.

Así y siguiendo la tabla 5, lo más valorado por los visitantes es la hospitalidad, la conservación del entorno, las actividades culturales y la restauración. Estos resultados de la valoración resaltan que esta actividad turística está muy relacionada con el turismo rural y que tiene componentes culturales importantes, como se ha concluido en otras investigaciones (Ruiz Guerra, 2010). En este sentido, y como ya se ha mencionado, en estas zonas de Andalucía donde se realiza esta actividad existen tres ciudades Patrimonio de la Humanidad, por esta razón, es importante reforzar el aspecto cultural del aceite de oliva como experiencia del turista, como ya ocurre con el turismo gastronómico (Lee y Scott, 2015). 
Por otro lado, el mejor servicio recibido en esta zona geográfica ha sido en las almazaras $(36,1 \%)$, seguido de la restauración $(34,9 \%)$, alojamiento $(24,6 \%)$ y la oferta complementaria $(4,4 \%)$. Por su parte, el 42,2\% está satisfecho con el viaje realizado, y un $41,7 \%$ muy satisfecho. En este sentido, el $85,7 \%$ repetiría el viaje. Así, se ha detectado asociación entre el nivel de satisfacción y las siguientes variables: sexo (Chicuadrado de Pearson $=10,224 ; \mathrm{p}=0,037$ ), nivel de la educación (Chi-cuadrado de Pearson $=56,565 ; \mathrm{p}=0,000)$ y país de origen (Chi-cuadrado de Pearson $=121,130 ;$ $\mathrm{p}=0,000)$. De esta manera, se ha encontrado asociación entre la satisfacción de los visitantes con el viaje y si repetirá el viaje a esta zona geográfica (Chi-cuadrado de Pearson $=21,743 ; \mathrm{p}=0,000)$. Por su parte, también se encontró asociación entre el nivel de renta y si repetiría el viaje (Chi-cuadrado de Pearson $=11.950 ; \mathrm{p}=0,018$ ). Otro aspecto estudiado en esta investigación es la relación entre la satisfacción del visitante con los diferentes variables del destino geográfico, analizado a través de una Correlación de Spearman (tabla 6).

\section{Tabla 6 \\ SATISFACCIÓN Y VALORACIONES A TRAVÉS DE LA CORRELACIÓN SPEARMAN}

\begin{tabular}{|r|c|r|c|}
\hline \multicolumn{1}{|c|}{ Aspecto a valorar } & Correlación & Aspecto a valorar & Correlación \\
\hline Almazara & $0,478^{* *}$ & Ecología & $0,373^{* *}$ \\
\hline Hospitalidad & $0,470^{* *}$ & Alojamiento & $0,361^{* *}$ \\
\hline Información & $0,461^{* *}$ & Limpieza & $0,358^{* *}$ \\
\hline Comunicaciones & $0,442^{* *}$ & Actividades culturales & $0,326^{* *}$ \\
\hline Seguridad ciudadana & $0,419^{* *}$ & Telecomunicaciones & $0,308^{* *}$ \\
\hline Restauración & $0,403^{* *}$ & Actividades deportivas & $0,305^{* *}$ \\
\hline Alimentación & $0,386^{* *}$ & Precio del viaje & $0,269^{* *}$ \\
\hline Conservación & $0,380^{* *}$ & Zona comercial & $0,265^{* *}$ \\
\hline
\end{tabular}

Fuente: Elaboración propia. **Correlación significativa al $1 \%$.

En la tabla 6 se puede observar que todas las variables analizadas muestran una significativa y positiva correlación en la satisfacción del turista, destacando la almazara, hospitalidad, información, comunicaciones y la seguridad ciudadana como las variables que tienen una mayor influencia sobre la satisfacción de los oleoturistas en este destino geográfico. Así, la calidad de un destino turístico y la satisfacción de los turistas forman parte de los elementos a tener en cuenta para mejorar la gestión y promoción de un área turística (Zabkar et al., 2010), y por tanto, se deben mejorar aquellos aspectos que no se tienen en cuenta para satisfacer las necesidades de los visitantes y potenciar aquellos otros que actualmente son tenidos en cuenta para obtener una mayor satisfacción en los turistas. Por último, esto afecta a la intención de repetir el viaje, puesto que elementos como la imagen de un destino o satisfacción de un viaje afectan positivamente a la lealtad del turista (Court y Lupton, 1997; Bigné et al., 2001). 


\section{CONCLUSIONES, LIMITACIONES Y FUTURAS LÍNEAS DE INVESTIGACIÓN}

La actividad turística forma parte de un sector económico que ha experimentado un cambio de tendencia, puesto que han aparecido turistas más exigentes con nuevas actitudes y necesidades, que tienen interés por la conservación de la naturaleza y el respeto hacia la cultura local. De esta manera, han aparecido nuevas formas de turismo encuadradas dentro de lo que se conoce como turismo temático, como es el caso del turismo gastronómico, enoturismo u oleoturismo. Este último se desarrolla en el medio rural donde se refleja una determinada cultura en relación al aceite de oliva. De esta manera, el objetivo de esta investigación es analizar el perfil sociodemográfico de los visitantes, las actividades que realizan, las motivaciones para realizar la visita, la valoraciones sobre elementos del destino y los niveles de satisfacción que tienen con el viaje en varias zonas geográficas de Andalucía donde se realiza el oleoturismo, encontrándose tales zonas en la provincia de Jaén y Córdoba, las dos principales zonas en producción de aceite de oliva en España. Para conseguir los objetivos de este estudio se realizó una encuesta a los oleoturistas.

Los principales resultados de esta investigación muestran que destacan las mujeres, con edades de 60 o más años, procedentes de España, jubilados, con una renta media-alta y con educación universitaria, como principal demanda turística en estas zonas geográficas de Andalucía. La mayoría de oleoturistas han visitado por primera vez estos destinos, destacando turistas frente a los excursionistas. La mayoría de los visitantes viajan con compañeros de trabajo, amigos o con pareja. La elección de estos destinos de oleoturismo proviene por experiencia propia o por la recomendación de amigos y familiares. Destaca el turismo cultural como la principal actividad que realizan los turistas durante el viaje, pudiendo deberse a que estas zonas están cerca de tres ciudades Patrimonio de la Humanidad (Córdoba, Úbeda y Baeza). Las principales motivaciones son visitar la zona geográfica, aprender sobre el mundo del aceite y comer y beber productos de la zona. Entre las principales valoraciones de los visitantes hacia el destino turístico destaca la hospitalidad, la conservación del entorno, las actividades culturales y la restauración. Como mejor servicio recibido en esta zona geográfica destacan las almazaras, seguido de la restauración, alojamiento y la oferta complementaria. La mayoría de visitantes están satisfechos con el viaje realizado o muy satisfecho, coincidiendo la mayoría con la posibilidad de repetir el viaje. Por último, cabe destacar que la almazara, hospitalidad, información, comunicaciones y la seguridad ciudadana son las variables que tienen una mayor influencia sobre la satisfacción de los oleoturistas en este destino geográfico.

La principal limitación de esta investigación procede de la corta temporalidad del estudio, debido a que se han recolectado datos solamente en los meses de primavera y principio del otoño, y, por tanto, no se valoran las motivaciones y satisfacción de los oleoturistas que llegan en otra época, pudiendo tener estos unas motivaciones diferentes. También, el estudio está enfocado solamente desde la demanda, dificultando la difusión de los descubrimientos a otros grupos de stakeholders. Como futuras líneas de investigación, se propone la realización de este estudio en otros destinos de oleoturismo de España, con la finalidad de comparar los resultados obtenidos en este trabajo con los de los otros destinos. Otra posible línea de investigación podría ser la realización de este mismo estudio en otros países competidores de esta zona geográfica (por ejemplo, 
Italia o Grecia), con la finalidad de comparar resultados sobre las valoraciones y opiniones de los oleoturistas. Por último, se podría analizar el oleoturismo a través de otros stakeholders diferentes a la demanda, como puede ser desde la perspectiva de la oferta o la administración pública.

\section{BIBLIOGRAFÍA}

ALONSO, A.D. (2010): «Olives, hospitality and tourism: a Western Australian perspective», British Food Journal, vol. CXII, n 1, pp. 55-68.

ALONSO, A.D. y KRAJSIC, V. (2013): «Food heritage down under: olive growers as Mediterranean "food ambassadors" », Journal of Heritage Tourism, vol. VIII, n ${ }^{\circ} 2-3$, pp. 158-171.

ALONSO, A.D. y NORTHCOTE, J. (2010): «The development of olive tourism in Western Australia: A case study of an emerging tourism industry», International Journal of Tourism Research, ${ }^{\circ}$ 12, pp. 696-708.

AMUQUANDOH, F.E. y ASAFO-ADJEI, R. (2013): «Traditional food preferences of tourists in Ghana», British Food Journal, vol. CXV, n 7, pp. 987-1002.

ARAMYAN, L.; ONDERSTEIJN, C.; VAN KOOTEN, O. y LANSINK, A.O. (2006): «Performance Indicators in Agri-food Productions Chains» en Ondersteijn, C.; Vijnands, J. H.; Huirne, R.B. y Van Kooten, O. (Eds.), «Quantifying the Agri-food Supply Chain», $\mathrm{n}^{\circ}$ 5, pp. 47-64. Holanda.

AYBAR, R. (2004). Proyecto Oleoturismo: una red europea para la promoción de la cultura del olivo. Jaén, Diputación Provincial de Jaén.

BIGNÉ, J.E.; SÁNCHEZ, M. I. y SÁNCHEZ, J. (2001): «Tourism Image, Evaluation Variables and After Purchase Behaviour: Inter-Relationship», Tourism Management, vol. XXII, nº 6, pp. 607-616.

BLACK, S.A. y PORTER, L.J. (1996): «Identification of the critical factors of TQM», Decision Science, vol. XXVII, $\mathrm{n}^{\circ} 1$, pp. 1-21.

BONIFACE, P. (2003): Tasting tourism: Travelling for food and drink. Burlington, Ashgate.

BRUNORI, G. y ROSSI, A. (2000): «Synergy and coherence through collective action: some insights from wine routes in Tuscany», Sociology Ruralis, vol. XL, no 4, pp. 409-423.

BRUWER, J. (2003): «South Africa wine routes: some perspectives on the wine tourism industry's structural dimensions and wine tourism product», Tourism Management, $\mathrm{n}^{\mathrm{o}}$ 24, pp. 423-435.

CAMPÓN-CERRO A.M.; DI-CLEMENTE E.; HERNÁNDEZ-MOGOLLÓN J.M.; DE SALVO P. y CALZATI, V. (2014): «Olive oil tourism in sourthern Europe: Proposals for tourism development of olive grove rural areas», Turismo \& Desenvolvimento, $\mathrm{n}^{\circ}$ 21-22, pp. 63-73.

CHANG, R.; KIVELA, J. y MAK, A. (2010): «Food preferences of Chinese tourists», Annals of Tourism Research, $n^{\circ}$ 9, pp. 371-386.

CLARK, G. y CHABREL, M. (2007): «Measuring integrated rural tourism», Tourism Geographies, $\mathrm{n}^{\circ}$ 9, pp. 371-386. 
COURT, B. y LUPTON, R.A. (1997): «Customer Portfolio Development: Modelling Destination Adopters, Inactives and Rejecters», Journal of Travel Research, vol. XXXVI, $\mathrm{n}^{\mathrm{o}} 1$, pp. 35-43.

CRACOLICI, M.F. y NIJKAMP, P. (2009): «The attractiveness and competitiveness of tourist destinations: A study of Southern Italian regions», Tourism Management, $\mathrm{n}^{\mathrm{o}}$ 30, pp. 336-344.

CONSEJO OLEÍCOLA INTERNACIONAL (2013): Producción de aceite de oliva, Madrid, Servicio de Publicaciones.

CORREIA, L.; PASSOS ASCENÇAO, M. y CHARTERS, S. (2004): «Wine routes in Portugal: a case study of the Bairrada Wine Route», Journal of Wine Research, vol. $\mathrm{XV}, \mathrm{n}^{\mathrm{o}} 1$, pp. 15-25.

DE SALVO, P.; HERNÁNDEZ MOGOLlÓN, J.M.; DI CLEMENTE, E. y CALZATI, V. (2013): «Territory, tourism and local products. The extra virgin oil's enhancement and promotion: A benchmarking Italy-Spain», Tourism and Hospitality Management, vol. XIX, n ${ }^{\circ} 1$, pp. 23-34.

DIPUTACIÓN DE JAEN (2015): «Jaén Selección 2015». Disponible en file://C:/Users/ Escuela\%20de\%20Graduados/Downloads/folleto-JS2015-espanol-BR.pdf

FINN M.; ELLIOTT-WHITE M. y WALTON M. (2000): Tourism and leisure research methods: Data collection, analysis and interpretation. Harlow, Pearson Education.

GETZ, D. (2000): Explore wine tourism, management, development and destinations. New York, Cognizant Communication Corporation.

HILLEL, D.; BELHASSEN, Y. y SHANI, A. (2013): «What makes a gastronomic destination attractive? Evidence from the Israeli Negev», Tourism Management, $\mathrm{n}^{\circ} 36, \mathrm{pp}$. 200-209.

HINOJOSA RODRÍGUEZ A.; PARRA-LÓPEZ C.; CARMONA-TORRES. C. y SAYADI S. (2014): «Protected designation of origin in the olive growing secor: adoption factors and godoness of practices in Andalusia, Spain», New Medit, vol. XIII, n 3, pp. 2-12.

KIVELA, J. y CROTTS, J. (2006): «Tourism and gastronomy: gastronomy's influence on how tourists experience a destination», Journal of Hospitality \& Tourism Research, vol. XXX, n ${ }^{\circ} 3$, pp. 354-377.

LEE K. H. y SCOTT N. (2015): «Food tourism reviewed using the paradigm funnel approach», Journal of Culinary Science \& Technology, n 13, pp. 95-115.

LÓPEZ-GUZMÁN T. y GONZÁLEZ-FERNÁNDEZ V. (2011): «Socioeconomic development in rural areas through the creation of tourist routes: An olive tourism approach in the Jaen province (Spain)», European Journal of Tourism, Hospitality and Recreation, vol. II, no 2 , pp. 5-18.

LÓPEZ-GUZMÁN, T. y SÁNCHEZ-CAÑIZARES, S. (2012): «Culinary tourism in Cordoba (Spain)», British Food Journal, vol. CXIV, n 2, pp. 168-179.

LÓPEZ-GUZMÁN, T.; RODRÍGUEZ GARCÍA y VIEIRA RODRÍGUEZ, A. (2013): «Revisión de la literatura científica sobre enoturismo en España», Cuadernos de Turismo, no 32 , pp. 171-188.

LÓPEZ-GUZMÁN T.; VIEIRA-RODRÍGUEZ A. y RODRÍGUEZ-GARCÍA J. (2014): «Profile and motivations of European tourists on the Sherry wine route of Spain», Tourism Management Perspectives, $\mathrm{n}^{\circ} 11, \mathrm{pp} .63-68$. 
McINTOSH, R.W.; GOELDNER, C. y RITCHIE, B. (1995): Tourism: Principles, practices, philosophies. New York, John Wiley \& Sons.

MOLINA MORENO V.; QUESADA RUBIO J.M. y RUIZ GUERRA I. (2011): «Potencial del oleoturismo como diversificación económica del sector cooperativo agrario: el caso español», Revista de Ciencias Sociales, vol. XVII, n 3, pp. 533-541.

MILLÁN VÁZQUEZ DE LA TORRE, M.G.; AGUDO GUTIÉRREZ, E.M. y MORALES FERNÁNDEZ, E. (2011): «Análisis de la oferta y la demanda de oleoturismo en el sur de España: un estudio de caso», Cuadernos de Desarrollo Rural, vol. VIII, nº 67 , pp. 181-202.

MILLÁN VÁZQUEZ DE LA TORRE, M.G.; ARJONA, J.M. y AMADOR, L. (2014): «A new market segment for olive oil: olive oil tourism in the south of Spain», Agricultural Sciences, vol. V, no 3, pp. 179-185.

MILLÁN VÁZQUEZ DE LA TORRE, M.G.; MORALES-FERNÁNDEZ, E.J. y PÉREZ NARANJO, L.M. (2012): «Análisis del turismo gastronómico en la provincia de Córdoba», Tourism \& Management Studies, $\mathrm{n}^{\circ}$ 8, pp. 78-87.

MILLÁN VÁZQUEZ DE LA TORRE, M.G.; MORALES FERNÁNDEZ, E. y PÉREZ NARANJO, L.M. (2014): «Turismo gastronómico, Denominaciones de Origen y desarrollo rural en Andalucía: situación actual», Boletín de la Asociación de Geógrafos Españoles, $\mathrm{n}^{\circ}$ 65, pp. 113-137.

MORAL CUADRA, S.; CAÑERO MORALES, P.; ORGAZ-AGÜERA, F. y LÓPEZGUZMÁN, T. (2014): «Una aproximación al oleoturismo en Andalucia, España», International Journal of World of Tourism, vol. I, n 2, pp. 31-43.

MORALES FERNÁNDEZ, E. (2012): «Denominaciones de Origen Protegidas (DOP) y turismo gastronómico: una relación simbiótica en Andalucía», Gran Tour, nº 6, pp. 101-121.

MURGADO, E.M. (2013): «Turning food into a gastronomic experience: olive oil tourism», Options Mediterranéennes, $\mathrm{n}^{\circ}$ 106, pp. 97-109.

NORTHCOTE J. y ALONSO A.D. (2011): «Factor underlying faro diversification: the case of Western Australia's olive farmers», Agriculture Human Values, n 28, pp. 237-246.

NUNNALLY, J. y BERNSTEIN, I. (1994): Psychometric Theory. New York, McGrawHill.

OKUMUS, B.; OKUMUS, F. y McKERCHER, B. (2007): «Incorporating local and international cuisines in the marketing of tourism destinations: the cases of Hong Kong and Turkey», Tourism Management, $\mathrm{n}^{\circ}$ 28, pp. 253-261.

PETRICK, J. y BACKMAN, S. (2002): «An examination of the construct of perceived value for the prediction of golf traveler's intentions to revisit», Journal of Travel Research, vol. XLI, n 1 , pp. 38-45.

PORIA Y.; BUTLER R. y AIREY D. (2003): «The core of heritage tourism», Annals of Tourism Research, $\mathrm{n}^{\circ}$ 30, pp. 238-354.

PULINA, M.; DETORRI, D. y PABA, A. (2006): «Life cycle of agrotouristic firms in Sardinia», Tourism Management, $\mathrm{n}^{\circ}$ 27, pp. 1006-1016.

QUAND, S. y WANG, N. (2004): «Towards a structural model of the tourist experience: an illustration from food experiences en tourism», Tourism Management, $\mathrm{n}^{\circ}$ 25, pp. 297-305. 
ROZIN, P. y VOLHMECKE, T. (1986): «Food likes and dislikes», Annual Review of Nutrition, $\mathrm{n}^{\circ}$ 6, pp. 433-456.

RUIZ GUERRA I. (2010): Análisis cuantitativo y cualitativo del significado del aceite de oliva. Una aproximación desde el punto de vista del consumidor. Granada, Servicios de Publicación de la Universidad de Granada.

RUIZ GUERRA, I.; MOLINA MORENO, V. y MARTÍN LÓPEZ, V.M. (2011): «El oleoturismo como atractivo turístico en el medio rural español», Papers de Turisme, $\mathrm{n}^{\mathrm{o}}$ 49-50, pp. 89-103.

SIM, R. (2009): «Food, place and authenticity: local food and the sustainable tourism experience», Journal of Sustainable Tourism, vol. XVII, $\mathrm{n}^{\circ}$ 3, pp. 321-336.

SMITH, S. y COSTELLO, C. (2009): «Culinary tourism: Satisfaction with a culinary event utilizing importance-performance grid analysis», Journal of Vacation Marketing, vol. XV, no 2 , pp. 99-110.

SOCHER, K. y TSCHURTSCHENTHALER, P. (1994): «Tourism and agriculture in Alpine regions», The Tourist Review, vol. XLIX, n 3, pp. 35-41.

TELFER, D. y WALL, G. (1996): «Linkages between tourism and food production», Annals of Tourism Research, vol. XXIII, nº 3, pp. 635-653.

TOMLJENOVIĆ, R. y GETZ, D. (2009): «Life-cycle stages in wine tourism development: a comparison of wine regions in Croatia», Tourism Review International, vol. XIII, $\mathrm{n}^{\circ} 1$, pp. 31-49.

TORRES, R. (2002): «Towards a better understanding of tourism and agriculture linkages in the Yucatan: tourist food consumption and preferences», Tourism Geographies, $\mathrm{n}^{\circ}$ 4, pp. 282-307.

SÁNCHEZ, A.V.; BUENO, N.P.; MEJÍA, M.Á.P. y LIGERO, F.R. (2012): «Turismo enológico: comportamiento del turista y percepción de la población residente», Papers de Turisme, $\mathrm{n}^{\circ}$ 43-44, 97-111.

VELIOKA, N.; MUROVA, O. y DODD, T. (2013): «Emerging wine market in the Dominican Republic: Consumer market analysis», Wine Economics and Policy, $\mathrm{n}^{\circ} 2$, pp. 76-84.

WOODLAND, M. y ACOTT, T. (2007): «Sustainability and local tourism branding in England's South Downs», Journal of Sustainable Tourism, no 15, pp. 715-734.

WOOLCOTT, D.; KAWASH, G. y SABRY, J. (1981): «Correlations of nutrition knowledge in Canadian businessmen», Journal of Nutrition Education, $\mathrm{n}^{\circ}$ 13, pp. 153-156.

ZABKAR, V.; BRENČIČ, M.M. y DMITROVIĆ, T. (2010): «Modelling perceived quality, visitor satisfaction and behavioural intentions at the destination level», Tourism Management, vol. XXXI, no 4, pp. 537-546. 
